\title{
Adnexal Mass in Adolescents And Their Management
}

\author{
Don Isaac ${ }^{1}, \mathrm{G}$ Nagarathna ${ }^{2}$ \\ Resident, Deparment Of OBG, Father Muller Medical College, Mangalore.
}

\begin{abstract}
Introduction: Adnexal lesions present in 2.6 per 1,00,000 of adolescents and is a source of concern for the patient and her parents. They usually present with pain abdomen and rarely mass per abdomen. Adnexal lesions in adolescents are usually benign but rarely can be malignant. Some lesions regress spontaneously but others which cause severe discomfort to the patient need to be managed surgically in which laproscopic surgery is considered beneficial.

Objectives: To evaluate adnexal lesions in adolescents and their management.

Materials And Methods: Prospective study done among adolescent girls in the age group 13 - 19 years presenting with adnexal lesion on evaluation at FATHER MULLERS MEDICAL COLLEGE HOSPITAL , MANGALORE between January 2015 to august 2016 were included in the study.

Results: 22 patients were diagnosed to have adnexal lesions, 16 of them underwent surgical procedure for their condition and 6 others underwent conservative management. 12 of them had a endometriotic cyst , 3 cases of serous cystadenoma, 4 of them ovarian torsion, 1 case each of fallopian tube torsion and dermoid cyst. No malignant adnexal lesion noted in this study.

Conclusion: There is an increase in endometriotic cyst among adolescents. An increase in surgical management of adnexal lesions was noted.Early diagnosis and management and fertility preservation can prevent future morbidity.
\end{abstract}

\section{Introduction}

Adnexal lesions in adolescents are usually rare, they are usually benign such as functional cysts which are common in the postpubertal period due to failure of the functional cyst to involute or due to persistence of ovarian follicles. Paraovarian and paratubal cysts are relatively uncommon in the adolescent age group but can be present. Paratubal cyst are of mesonephric origin and paraovarian cysts of Wolffian origin. They may present with abdominal pain and can cause torsion or rupture. These cysts require conservative management and if the size is large will need surgical management. Some of the adnexal lesions present as an emergency and need immediate management such as ovarian torsion. Ovarian endometriosis is now increasingly seen among adolescent girls resulting in chronic pelvic pain, severe dysmenorrhea . Endometriotic cysts have a detrimental effect on the ovarian reserve as the diagnosis is delayed. Malignant adnexal lesions accounts about $9-11 \%$ of the cases. They usually present with pain abdomen, heavy menstrual bleeding , menstrual irregularity and rarely mass per abdomen. Diagnosis of such lesions causes anxiety and concern among the patients and their parents and need to be managed with care and dilligence.

\section{Methods}

The study was conducted in Father Mullers Medical college from January 2015 to June 2016. It was a prospective study done among 22 adolescents who presented with features of pain abdomen and /or mass per abdomen and thorough history was elicited and on general physical examination ultrasound and other evaluation was found to have an adnexal lesion and were included in the study, ultrasound abdomen was done . Relevant blood investigations were done and their management and follow up done.

\section{Results}

The total number of adolescents who were evaluated were 22 , out of which 16 of them underwent surgical procedure namely laproscopic approach for their condition Demographic profile- the age of the study group ranged from 13 to 19 years. The mean age of the study group was 16.27 years. The mean age of menarche was 12.5 years among this group. Symptoms at presentation - Adnexal lesions present in varied ways the main complaint they presented in this study was pain abdomen, menorrhagia, irregular cycles, mass per abdomen. Patients with adnexal torsion present with severe pain abdomen which is sudden in onset associated with nausea, vomiting. Patients with endometriotic cysts, benign ovarian cyst had a longer duration of complaints. Their mean duration of complaints was 3.6 months. The patients presenting with ovarian torsion had a shorter duration of complaints, their mean duration of complaints in this study was 4 days. 


\begin{tabular}{|l|l|l|}
\hline Symptoms & No of cases & Percentage \\
\hline Pain abdomen & 12 & $54.5 \%$ \\
\hline Severe dysmenorrhea & 5 & $22.8 \%$ \\
\hline Menorrhagia & 4 & $18.2 \%$ \\
\hline Mass per abdomen & 1 & $4.5 \%$ \\
\hline TOTAL & 22 & $100 \%$ \\
\hline
\end{tabular}

In this study the major complaint in $54 \%$ of the patient was pain abdomen followed by menorrhagia in $18.1 \%$, severe dysmenorrhea in $13.4 \%$ and mass per abdomen in $4.5 \%$.

Usg Findings

\begin{tabular}{|l|l|l|}
\hline USG diagnosis & No of cases & Percentage \\
\hline Haemorrhagic/endometriotic cyst & 11 & $50 \%$ \\
\hline Simple ovarian cyst/ paraovarian cyst & 5 & $22.7 \%$ \\
\hline Ovarian torsion & 4 & $18.1 \%$ \\
\hline Dermoid cyst & 1 & $4.5 \%$ \\
\hline Fallopian tube torsion & 1 & $4.5 \%$ \\
\hline
\end{tabular}

On ultrasound haemorrhagic/ endometriotic cyst were most commonly seen in $11(50 \%)$ patients Bilateral endometriotic lesions was seen in 4 patients, followed by features of simple ovarian cyst in $5(22.7 \%)$ of cases . Ovarian torsion was picked up on ultrasound in 4(18.1\%) of cases followed by 1 patient each of dermoid cyst and fallopian tube torsion. Among patients with endometriosis the maximum value of CA 125 was 39.15 , and ranged from 8.75 to39.15

\begin{tabular}{|l|l|l|}
\hline Management \\
\hline Management & Number & Percentage \\
\hline Conservative & 6 & $27.2 \%$ \\
\hline Laproscopic cystectomy Laproscopic Salpingo- & 11 & $50 \%$ \\
\hline $\begin{array}{l}\text { Unilateral } \\
\text { oopherectomy }\end{array}$ & 4 & $18.1 \%$ \\
\hline Laproscopic salpingectomy & 1 & $4.5 \%$ \\
\hline
\end{tabular}

Among the 22 patients 16(72.7\%)underwent surgical management for the adnexal lesion. Patients diagnosed to have endometriotic cyst who were symptomatic and size larger than $5 \mathrm{cms}$ underwent laproscopic cystectomy and the 4 patients with bilateral lesions underwent cyst deroofing on the smaller lesion. The largest ovarian lesion measured $10 \times 7 \mathrm{cms}$. Patients with ovarian torsion and dermoid cyst underwent unilateral laproscopic salpingo-oopherectomy and the 1 patient with fallopian tube torsion underwent laproscopic salpingectomy. Conservative treatment was done in 6(27.3\%)patients, they were treated by analgesics and OCP tablets.

\begin{tabular}{|c|c|}
\hline \multicolumn{2}{|c|}{ Histopathological diagnosis } \\
\hline HPE Diagnosis & Number \\
\hline Haemorrhagic cyst & 7 \\
\hline Benign serous cystadenoma & 2 \\
\hline Serous cystadenofibroma & 1 \\
\hline Benign mucinous cystadenoma & 1 \\
\hline Dermoid cyst & 1 \\
\hline
\end{tabular}

After surgery the specimen was subjected to histopathological examination. The specimens of endometriotic cyst was reported as haemorrhagic cyst. Case of paraovarian cyst HPE came as serous cystadenofibroma. No cases of malignant adnexal lesion was noted in this study.

\section{Discussion}

Though majority of the adnexal lesions are benign and benefit from conservative management because surgical procedures can affect ovarian function. In this Study 6 of the patients underwent conservative management , 4 of them had a haemorrhagic cyst size less than $5 \mathrm{cms}$ and 2 of them with features of simple cyst size less than $6 \mathrm{cms}$ and were followed up. Patients with endometriotic cyst larger than $5 \mathrm{cms}$ and who were symptomatic underwent laproscopic cystectomy.The size of their Endometriotic cysts if they are larger than 4 cms warrant surgery as endometriosis is assumed to be a progressive disease, the ACOG recommends the early diagnosis and treatment in the adolescents. there is an increase in incidence of endometriosis in adolescents as seen in this study $50 \%$ of adnexal lesions were due to endometriotic cyst. Adolescents presenting with dysmenorrhea and menstrual complaints should be investigated for endometriosis. Although most women with endometriosis do report the onset of symptoms during adolescence but diagnosis is often delayed, this delay 
may decrease their reproductive potential . Therefore early identification and treatment of endometriosis may resolve pain, prevent disease progression and organ damage and preserve fertility.

Ovarian torsion needs immediate diagnosis, they present with severe pain abdomen, colour Doppler can be used to assess vascularity and immediate surgical detorsion needs to be done. In this study all cases of torsion underwent laproscopic salpingo-oopherectomy.

Therefore a timely and pragmatic approach is required while managing adnexal lesions in adolescents to achieve optimum outcome and fertility of the patients should be preserved to the maximum extent.

\section{References}

[1]. Dotters-Katz SK, James AH, Jaffe TA (2014) Paratubal/Paraovarian Masses: A Study of Surgical and Non-Surgical Outcomes. Med J Obstet Gynecol 2(1): 1019.

[2]. Gordts S, Puttemans P, Gordts S, Brosens I. Ovarian endometrioma in the adolescent: a plea for early-stage diagnosis and full surgical treatment. Gynecol Surg. 2015;12(1):21-30.

[3]. Laufer, M. and Goldstein, D. Benign and malignant ovarian masses. Pediatric and adolescent gynecology. Lippincott Williams \& Wilkins, Philadelphia,2000, 685-728.

[4]. Kanizsai, B., Orley, J., Szigetvari, I. and Doszpod, J. (1998) Ovarian cysts in children and adolescents: Their occurrence, behavior, and management. Journal of Pediatric and Adolescent Gynecology, 11, 85-8.

[5]. Pfeifer, S, Gosman, G Evaluation of adnexal masses in adolescents. Pediatric Clinics of North America,1999;46,573-92

[6]. Spinelli C, Buti I, Pucci V, Liserre J, Alberti E, et al,Adnexal torsion in children and adolescents :new trends to conservative surgical approach-Our experience and review of literature. Gynecol Endocrinol2012,29:54-8.

[7]. Hassan E, Creatsas G, Deligeorolgou E, Michalas S,Ovarian tumors during childhood and adolescence. A clinicopathological study. Eur J Gynaecol Oncol 2012,20:124-6. 